\title{
Pressure gain combustion
}

\author{
E. J. Gutmark ${ }^{1}$ (I)
}

Published online: 2 December 2021

(c) The Author(s), under exclusive licence to Springer-Verlag GmbH Germany, part of Springer Nature 2021

Pressure gain combustion (PGC) is an unsteady and periodic process whereby a net increase in stagnation pressure is achieved by gas expansion in a constrained heat release environment. Higher work extraction is then available by expansion of the gases to the initial pressure. Devices based on this concept include resonant pulse combustor (RPC) or pulse jets, pulsed detonation combustors/engines (PDC or $\mathrm{PDE}$ ), and the more recent focus of research, the rotating detonation combustor/engine (RDC/RDE). The latter fact is also evident from the focus of most of the papers that are included in the current thematic issue.

RDCs have potential advantages over PDEs, RPCs, and deflagrative combustors in terms of high power density [1], no moving parts, no need to regulate periodic ignition and reactants' injection, high operating frequency, and steadier exit flow profile [2], making it more adaptable to turbine integration with high pressure gain, and low emissions [3]. However, despite considerable progress made on the different aspects of RDC physics, design, and operation, there are still technical challenges to resolve, and flow and combustion physics to be studied, so that it can become a practical propulsion or power generation system. These topics include efficient injection and mixing of homogeneous and heterogeneous (liquid and solid fuels) reactants, initiation and sustaining stable detonation, control of instabilities and operational modes, operation under back-pressurized conditions, characterization of internal and exit flow fields, demonstration of pressure gain, impact of the combustor interaction with upstream and downstream components, and management of the high heat and mechanical loading. Some of the technical challenges enumerated above are the subject of the investigations reported in the papers that are included in this thematic issue.

Manuscripts for the thematic issue on pressure gain combustion were solicited from the papers that were presented at

$凶$ E. J. Gutmark

gutmarej@ucmail.uc.edu

1 Department of Aerospace Engineering, University of Cincinnati, P.O. Box 210070, Cincinnati, OH 45221-0070, USA the 27th International Colloquium on the Dynamics of Explosions and Reactive Systems (ICDERS), which was held in Beijing, China, on July 28-August 2, 2019. The growing interest in pressure gain combustion was clearly demonstrated in the program of this colloquium with two plenary lectures, eight sessions, 29 lectures, and 25 posters, all dedicated to this topic. Submissions to this thematic issue were subjected to a rigorous peer-reviewed process following the established standards and policies of the Shock Waves Journal. Each paper was reviewed by at least three internationally recognized experts in the field. Twenty-four manuscripts were received in response to the thematic issue announcement, of which ten papers were rejected after peer review, or withdrawn by the authors following a "major revision" decision. The remaining 14 papers were eventually accepted upon up to four revisions and after a consensus was reached by the referees and the editors. Thus, the rejection rate for the issue is $42 \%$, which is one of the indicators of the high standards applied when preparing the issue.

The 14 papers included in this thematic issue can be grouped into three categories. The first group includes five contributions on the effects of geometry, reactants, and injection parameters on the operational mode of the RDC, its stability, and performance. Bohon et al. demonstrated the use of dynamic mode decomposition (DMD) to analyze the dynamic behavior of detonation waves in an RDC and capture the dominant features of the combustion. The analysis was performed on high-speed images of the detonations that were captured from the aft end of the combustor. The analysis was applied to a single rotating wave with a weak secondary counter-rotating wave and to two counter-rotating waves of equal speed and strength. They identified the limitations of the technique due to the presence of standing waves.

The impact of the number of detonation waves rotating within a disk-shaped annular gap on thrust and specific impulse was studied numerically for different levels of stagnation pressure in the propane-air premixed supply chamber (Levin et al.). The single-step combustion kinetics computations showed small effect on thrust and increased specific impulse with the number of waves. Subcritical stagnation 
pressure did not initiate detonations, and supercritical conditions varied the shock-wave structures with an increased pressure. The detonation initiator enabled to determine the direction of rotation and the number of corotating waves.

A reduced-order model was developed to describe the operation of an RDC for given engine conditions and geometry (Connolly-Boutin et al.). The model includes several geometrical assumptions: the presence of at least one detonation wave, a channel width of about three detonation cell widths, and a detonation wave height of about five channel widths. The model is then used to predict minimum injection rate of $\mathrm{H}_{2} / \mathrm{O}_{2}$ and $\mathrm{C}_{2} \mathrm{H}_{4} / \mathrm{O}_{2}$ reactants to obtain stable single detonation wave operation.

The impact of counter-rotating secondary acoustic and detonation waves on the primary detonation wave was investigated experimentally. Chacon et al. show that the interaction results in a substantial increase in pressure rise across the wave but suppresses the heat release due to the secondary wave impact on the reactants' supply.

The effect of the annular channel shape of an RDC was investigated numerically by Niyasdeen et al. The shapes included circular geometries of different radii of curvature, race-track, box, and star. The radius of curvature produced a flow compression effect that overdrove the wave speed beyond the $\mathrm{C}-\mathrm{J}$ speed. Detonation could not be stabilized below a critical radius of curvature. Stable detonation was observed in the linear section of the race-track and became destabilized in the curved sections. The box- and star-shaped configurations showed stable detonation in the high radius of curvature sections, but detonation did not stabilize in the star-shaped.

The second set of six papers is concerned with reactants injection of gaseous and liquid fuels. Operating RDEs on liquid fuel are necessary if the engine is to be used for practical propulsion applications such as gas turbines. A new method of utilizing liquid fuel (extraction gasoline and Jet-A) is presented by Wolański et al. The fuel is prevaporized by mixing it with hot air $\left(100^{\circ} \mathrm{C}\right)$ in the supply chamber while keeping the mixture above the rich flammability limit to prevent ignition before entering the combustor. Successful detonations were achieved for equivalent ratios between 0.95 and 1.25 and flow rates of $286-391 \mathrm{~g} / \mathrm{s}$. Peak detonation pressure measured was $1.5-2$ bars.

The interaction between a pressurized combustion chamber and liquid fuel injectors was tested using nine different injector geometries (Celebi et al.). The detonation wave was initiated by ethylene/oxygen reactants, and water was used to detect back pressure impact. The injectors differed by their length, injection angle, and taper angle. The cycle of backflow and refill was documented using high-speed videos. Stronger detonations produced more backflow but shorter refill time. Shorter injectors mitigate backflow by higher supply pressures. The injection angle had minimum effect but tapering increased response time and reduced sensitivity to supply and combustion pressures.

In a numerical study, Ren et al. investigated the impact of unsteady equivalence ratio of pre-vaporized kerosene-air on an oblique detonation wave. The unsteadiness induced unsteady transition from deflagration to detonation and deformed the wave structure. In the range of mild fluctuations, increasing amplitude resulted in the formation of triple points and disturbed initiation. High-amplitude fluctuations destabilized the detonation wave and formed strong transverse detonation waves. However, the detonation wave could be stabilized after a transitional readjustment.

Increasing flow rate of reactants into an annular RDC was studied experimentally and numerically and showed a resulting increase in wave velocity (Sato et al.). Equivalence ratio was varied between 0.75 and 1.25 , and two flow rates were considered. It was shown that the increase in supply pressure that allows for increased flow rate reduced the recovery time of the injectors due to steadier reactant supply and better mixing, thus affecting the deflagration zones characteristic of a non-premixed operation of the RDC.

An effective injector area that is defined as the ratio between the plenum pressure during operation to preignition level was used to assess the impact of the pressure balance between the RDC combustor and the supply plenum (Goto et al.). Two triplet injectors of different diameters were tested with methane, ethylene, and oxygen. The effective injector area decreased proportionally to the ratio of combustor to plenum pressure. Smaller injector resulted in increased wave speed with no effect on combustion pressure and specific impulse.

Numerical study of an annular RDC was performed for different equivalence ratios and with the addition of air or oxygen through lateral walls (Mikhalchenko et al.). The impact of these parameters on thrust and specific impulse is reported. Maximum thrust was obtained for rich mixtures, while specific impulse was highest at lean conditions. Both performance measures increased for rich mixture with added air/oxygen. At stoichiometric and lean conditions, only specific impulse increases. With oxygen addition, single detonation wave occurs. Air addition reduces the number of waves and causes unsteadiness in the detonation wave.

The third group includes two papers that describe detonation initiation of a pulse detonation engine by shock wave focusing (Bengoechea et al.). Shock focusing is achieved by a converging-diverging nozzle. One of the papers is a numerical study describing the DDT process. In the second paper, an adjoint approach is applied to optimize the geometry of the C-D nozzle and thus reduce the strength of the shock wave necessary for initiation of detonation. 
Finally, Wolański reviews RDC research in Poland and describes the activities to apply this technology to turbine engines, rockets, ramjets, and combined cycles.

This thematic issue highlights the increased recent interest in pressure gain combustion in general and especially in RDCs and will encourage further scientific contributions and discussions on the fundamental physics and on the applications of this new and exciting topic.

Acknowledgements I wish to thank the authors who submitted their work to this issue and revised their manuscripts to be in line with the high-quality standards of the journal. I also like to acknowledge the efforts by all referees to review these papers and make it possible to publish this thematic issue. Special thanks to Evgeny Timofeev and Harald Kleine who helped me with the organization of this issue.

\section{References}

1. Wolański, P.: Detonative propulsion. Proc. Combust. Inst. 34, 125158 (2013). https://doi.org/10.1016/j.proci.2012.10.005

2. Yi, T.-H., Lou, J., Turangan, C., Choi, J.-Y., Wolanski, P.: Propulsive performance of a continuously rotating detonation engine. J. Propuls. Power 27, 171-181 (2011). https://doi.org/10.2514/1. 46686

3. Anand, V., Gutmark, E.: Rotating detonation combustors and their similarities to rocket instabilities. Prog. Energy Combust. Sci. 73, 182-234 (2019). https://doi.org/10.1016/j.pecs.2019.04.001

Publisher's Note Springer Nature remains neutral with regard to jurisdictional claims in published maps and institutional affiliations. 